\title{
Fast Infrared Imaging for the Early Detection of Cancer
}

Thomas Tague and Stephan Luettjohann

Bruker Corporation, Billerica, Massachusetts, United States

Early detection and accuracy are key elements to the successful characterization of cancer. The earlier the detection is conducted, the more successful the outcomes. Infrared (IR) spectroscopy is emerging technique in the detection of cancer. ${ }^{1,2}$ Most importantly, changes in cell biochemistry precede any morphological manifestations allowing for earlier detection of disease. This would include not only the detection of significantly diseased tissue, but also early lesions and tissue dysplasia. Traditionally, light microscopy has been a key element of the analysis of tissue samples by pathologists. The interpretation of tissue and tumors containing borderline or indeterminant changes can lead to a false negative assessment. FTIR has been used successfully to assess the presence of cancer in the cervix ${ }^{3,4}$, colon ${ }^{5}$, pancreas $^{6}$, and other tissue ${ }^{2}$. Full field IR imaging provides an alternative that can provide a non-subjective analysis in the early detection of cancer over a large area. In particular, no tissue fixation or staining is required and the cost per test can be inexpensive. Until recently, fast IR imaging over the full spectral domain was somewhat limited in analyzing samples over the desired area of interest ( several to many square $\mathrm{mm}){ }^{7}$ Other approaches either limit the spectral region of the data collection of are somewhat slow in the data collection. Additionally, tissue sample analysis by IR is a noise limited experiment, meaning that the signal is very strong and brighter sources of light provide no benefit. Virtually all brighter IR sources have more nascent noise than traditionally utilized SiC sources thereby reducing the limits of detection.

For this investigation, a LumosII (Bruker Corporation) was utilized to conduct fast full-field FPA analysis of various relevant tissue samples over the full middle IR region. Figure 1 shows an infrared image of a mouse colon. The data was collected over a $5.12 \mathrm{~mm}^{2}$ area yielding 1.05 million spectra in total with a pixel resolution of $5 \mu \mathrm{m}$. A SiC IR source was utilized as well as a 32x32 pixel focal-plane array PVMCT detector. The spectral range was $5000-750 \mathrm{~cm}^{-1}$ collected at a spectral resolution of $4 \mathrm{~cm}^{-1}$. The total acquisition time was 35 minutes. The benefits of utilizing the infrared spectrum are clear in that the presence and distribution of each component of the colon are illustrated to facilitate the assessment of the tissue. Each molecule has its own "fingerprint" signature in the middle infrared region of the spectrum (400-4,000 $\left.\mathrm{cm}^{-1}\right)$. Because of this high degree of specificity, small changes to tissue can be detected. Examples will also be shown of the potential for kidney and bone tissue analysis in the detection and characterization of cancer. 

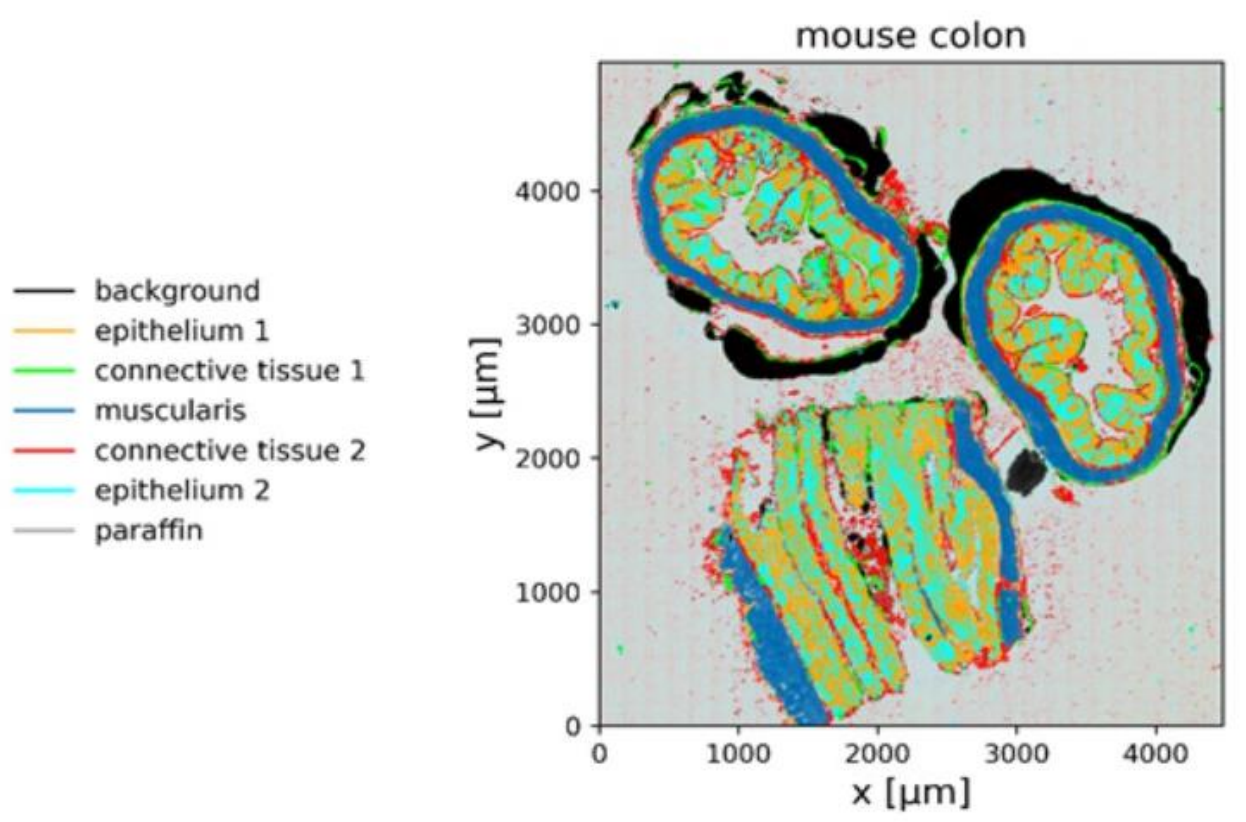

Figure 1. A FTIR image of a mouse colon collected with a LumosII in 35minutes at $4 \mathrm{~cm}^{-1}$ spectral resolution.

\section{References}

1. Othman, N.H., El-Tawil, S.G., "FTIR Spectroscopy - A new technique in cancer diagnosis", Journal of Lymphology and Oncology, 2008.

2. Lyman, D.J. and Fay, S.G., "The effect of breast cancer on the Fourier transform infrared attenuated total reflection spectra of human hair", ecancer 2014, 8:405.

3. Cohenford, M.A., Godwin, T.A., Cahn, F., et al, "Infrared spectroscopy of normal and abnormal cervical smears: evaluation of principle component analysis", Gynecol. Oncol., 1997, 66: 59.

4. Cohenford, M.A. and Rigas, B., "Cytologically normal cells from neoplastic cervical samples display extensive structural abnormalities on IR spectroscopy: implications for tumor biology”, Proc. Natl. Acad. Sci., 1998, 95:15327.

5. Salman, A., Argov, S., Ramesh, J., et al, "FT-IR microscopic characterization of normal and malignant human colon tissues", Cell. Mol. Biol., 2001, 47:OLI59.

6. Chen, Y.J., Cheng, Y.D., Liu, H.Y., et al, "Observation of biological imaging changes in human pancreatic cancer tissue using Fourier-transform infrared microspectroscopy”, Chang Gung Med J, 2006, 29:518.

7. https://www.agilent.com/en/products/ldir-chemical-imaging/ldir-chemical-imaging-system/8700-ldirchemical-imaging-system 